\title{
Valores normativos para determinar un pie plano o cavo
}

\author{
Normative values to determine a flatfoot or cavus foot \\ Carles Escalona-Marfila,b, Thomas McPoilc, Luis Enrique Roche-Seruendo ${ }^{d}$, \\ Xavier Dídac Ortas-Deunosajut ${ }^{a}$, Albert Pérez-Bellmunt ${ }^{e}$ y Bill Vicenzino ${ }^{f}$
}

aUniversitat de Vic-Universitat Central de Catalunya, Spain. ${ }^{b}$ EUSES - University of Girona, Spain. ${ }^{c}$ School of Physical Therapy, Regis University,

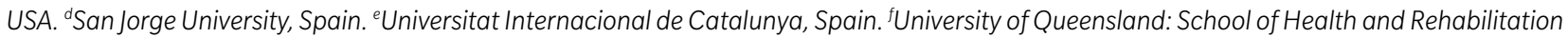
Sciences: Physiotherapy, Australia.

\section{Palabras clave:}

Pie plano, biomecánica, articulaciones del pie, hipermovilidad exploración física.

\section{Resumen}

Introducción: La altura del arco medial del pie es una característica de gran importancia, no solo por su relación con la probabilidad de sufrir lesiones y con la eficacia de sus tratamientos, sino también por ser un motivo de consulta clínica habitual tanto en población infantil como adulta. El diagnóstico y las decisiones terapéuticas deben basarse en la evidencia de su eficacia y, a su vez, en mediciones objetivas, válidas y fiables. Los objetivos de este artículo son dos: 1) describir un instrumento de medición de la postura y la movilidad del pie y su protocolo y 2) proponer unos valores de referencia basados en sus medias y puntos de corte.

Pacientes y métodos: Se describe el protocolo de exploración del pie mediante la plataforma de medición antropométrica del pie (p-MAP), con las que se obtienen la altura del arco dorsal y la anchura del mediopié, así como la movilidad en ambos planos y global del pie. Se proporcionan valores de referencia y puntos de corte para categorizar los pies en base a 1525 sujetos.

Resultados: Los valores de media (desviación típica) para la altura del arco dorsal son de $64.5 \mathrm{~mm}$ (5.6) en carga y $74.2 \mathrm{~mm}$ (5.0) en descarga, mientras que para la anchura del mediopié son de $83.5 \mathrm{~mm}$ (6.3) en carga y $75 \mathrm{~mm}$ (4.9) en descarga. El Índice de la Altura del Arco (IAA), que no tiene unidades por lo que se pueden comparar poblaciones con tamaños de pies muy distintas, presenta un valor de 0.251 (0.02). Los valores de movilidad tienen una media (desviación típica) de 11.31 mm (2.37) para la altura del arco doral, $9.51 \mathrm{~mm}$ (2.94) para la anchura del mediopié y 10.80 (2.80) para la movilidad global. Se proporcionan también los valores \pm 1 y \pm 1.5 desviaciones típicas y los IC $95 \%$.

Conclusiones: Se ha descrito la p-Map y el protocolo de medición que permite cuantificar de forma fiable y válida la postura y el grado de movilidad del pie. Se aportan los valores medios y unos puntos de corte en base a 1 y 1.5 desviaciones típicas que permiten establecer los valores de normalidad en base a 1525 sujetos.

Keywords:

Flatfoot, biomechanics, foot joints,

hypermobility, physical examination.

\begin{abstract}
Introduction: The medial arch height is a foot feature of great importance, not only because of its relationship with the likelihood of suffering injuries and the effectiveness of their treatments, but also because it is a common clinical reason for consultation in both children and adults. Podiatric diagnosis and therapeutic decisions should be evidence based, according to their effectiveness, and sustained on objective, valid and reliable measurements. The objectives of this article are two: 1) to describe an instrument for measuring the foot posture and mobility and its protocol and 2) to propose reference values based on their means and cut points.

Patients and methods: The protocol for the exploration of the foot is described by means of the foot anthropometric measurement platform (FAP or p-MAP in Spanish). The dorsal arch height and the midfoot width were measured, as well as the foot mobility magnitude. Reference and cut-off points are displayed, based on 1525 subjects.

Results: Mean (Standard Deviation -SD) values for Dorsal Arch Height are $64.5 \mathrm{~mm}$ (5.6) when the subject is weightbearing, and $74.2 \mathrm{~mm}$ (5.0) in non-weightbearing; midfoot width values are $83.5 \mathrm{~mm}$ (6.3) when weightbearing, and $75 \mathrm{~mm}$ (4.9) when non-weightbearing. The Arch Height Index (AHI, or Índice de la Altura del Arco (IAA)) has no units, which allows to compare samples with different foot lengths; its mean (SD) is 0.251 (0.02). The foot mobility values are $11.31 \mathrm{~mm}$ (2.37) -mean (standard error)- for the dorsal arch height, 9.51 (2.94) for the midfoot width, and 10.80 (2.80) for the overall mobility. Values for \pm 1 and \pm 1.5 standard deviations and IC95\% are also provided.

Conclusions: The protocol for the application of the anthropometric foot measurement platform ( $p$-MAP) is described for the first time in Spanish. This exploration methodology allows to reliably and validly quantify the posture and the degree of global mobility of the foot. Likewise, a categorization is proposed based on more than 1525 subjects.
\end{abstract}




\section{INTRODUCCIÓN}

El rendimiento deportivo y el riesgo de sufrir lesiones parecen estar relacionadas con la postura más o menos aplanada (considerada también por algunos autores como pronada) y con la rigidez del pie y del tobillo ${ }^{1}$. Un claro ejemplo es la fascitis plantar, que parece estar biomecánicamente relacionada con una falta de movilidad del tobillo²; otro ejemplo, a mayor distancia anatómica, es la dorsiflexión del tobillo, que parece estar relacionada con la prevención de lesiones del ligamento cruzado anterior de la rodilla ${ }^{3}$. Otro elemento importante es la relación entre el aplanamiento o pronación del pie con parámetros espaciotemporales durante la carrera ${ }^{4}$. Por lo tanto, se hace cada vez más evidente que la postura y la movilidad del pie están relacionadas con la probabilidad de sufrir determinadas lesiones ${ }^{5-8}$ y con la eficacia de determinadas terapias para el tratamiento de afecciones músculo-esqueléticas ${ }^{9,10}$.

Una medición válida y fiable de la postura del pie es imprescindible para un correcto diagnóstico y un plan de tratamiento clínico basado en la evidencia científica que asegure la mejor alternativa posible. Esta relación entre los ámbitos clínico y científico se hace cada vez más evidente ${ }^{11}$.

Existen multitud de pruebas para caracterizar la postura del pie, con distintos niveles de fiabilidad y validez. Una de las más habituales por la escasa infraestructura necesaria y su fiabilidad (de más del $80 \%$ ), entre otros motivos, es el Foot Posture Index (FPI o IPP, por su traducción al español como índice postural del pie), una medida global de la supinación o pronación del pie en posición estática ${ }^{4,5}$. Sin embargo, además de esta medida global, es interesante el análisis de parámetros individuales, como la altura del arco (o arco longitudinal medial [ALM]), que también puede ser medido de forma fiable, como comprobaron Williams y McClay ${ }^{12}$. En la práctica clínica, durante muchos años se han clasificado los pies en planos, neutros o cavos en función de la altura del ALM a través de la observación directa (antropometría) o mediante la huella plantar, con la ayuda de un podoscopio, o una plataforma de presiones, aunque se debe tener en cuenta que no representan la misma información ${ }^{13}$.

Por otro lado, el análisis del retropié también ha sido utilizado durante muchos años para clasificar el pie como varo o valgo a través de la observación directa. Sin embargo, la valoración del mediopié está demostrando ser un elemento clave para la comprensión de la biomecánica y la patología del pie, así como del resto de la extremidad inferior. Varios estudios demuestran que, durante la marcha, la cantidad de movimiento del navicular es mayor que la del calcáneo ${ }^{14}$, que la movilidad en la articulación talo-navicular es mayor que en la articulación subtalar, y que el movimiento entre el cuneiforme medial y el navicular llega hasta los $10^{\circ}$, tanto en los planos sagital como frontal ${ }^{15}$.

Además de la posición que ocupan los huesos en el espacio, que determina la postura del pie, su exploración física se complementa con la valoración clínica y manual de su grado de movilidad o rigidez. Una forma de cuantificar la movilidad del pie es calculando la diferencia que presenta un valor de postura del pie entre carga y descarga, o entre el pie relajado y neutralizado ${ }^{16,17}$.

En la práctica clínica, no siempre se tiene acceso a costosos métodos de valoración de la postura y la movilidad del pie, como plataformas de presiones, sistemas de análisis 3D de la marcha o la tomografía computerizada en carga. Sin embargo, existen herramientas de bajo coste, pero de elevada validez y fiabilidad. Entre estas herramientas se encuentra la plataforma de medición antropométrica del pie (p-MAP), que ha sido utilizada previamente y descrita a nivel internacional pero no en lengua española, con el nombre de Foot Assessment Platform (FAP). Además, las herramientas de medición deberían seguir un protocolo claro de utilización para que los resultados sean comparables, ya que un cambio en la posición del sujeto puede modificar los resultados obtenidos y, por lo tanto, inducir a error ${ }^{18}$.

El objetivo del presente trabajo es doble: 1) describir un protocolo para medir de forma fiable la postura y la movilidad del pie mediante el uso de la p-MAP, y 2) proporcionar unos valores de referencia para describir los pies planos o cavos.

\section{PACIENTES Y MÉTODOS}

La descripción del protocolo se basa en una revisión bibliográfica previa y en la experiencia de los autores, entre los cuales se encuentran los impulsores de la p-MAP. Es la primera publicación científica que describe este protocolo en español.

Para obtener los valores de referencia, se han recopilado los principales artículos encontrados en la bibliografía para cada medida de movilidad de pie y se han calculado diversos valores estadísticos.

Dados los diversos objetivos de cada artículo, los test y las variables estadísticas se pueden encontrar de distinta forma en cada uno de ellos. Los criterios seguidos han sido los siguientes: en los artículos con más de un observador, se han utilizado los valores del observador con mayor fiabilidad; para los artículos separados por hombres y mujeres, se han calculado las medias totales ponderadas al tamaño de la muestra respectiva; para los artículos separados por pie derecho y pie izquierdo, se ha calculado la media entre ambos.

\section{Plataforma de medición antropométrica del pie (p-MAP)}

El material propuesto para realizar los test es la Plataforma de Medición Antropométrica del Pie ( $p$-MAP, o Foot Assessment Platform, FAP en inglés) ${ }^{16}$. Consta de una base en material plástico con dos referencias para el talón y la cabeza del primer metatarsiano: unas "copas" de forma semicircular separadas 15,24 cm para situar los talones y unos topes móviles en la zona medial del pie para las cabezas de los primeros metatarsianos. Entre los dos pies se encuentra una guía mili- 


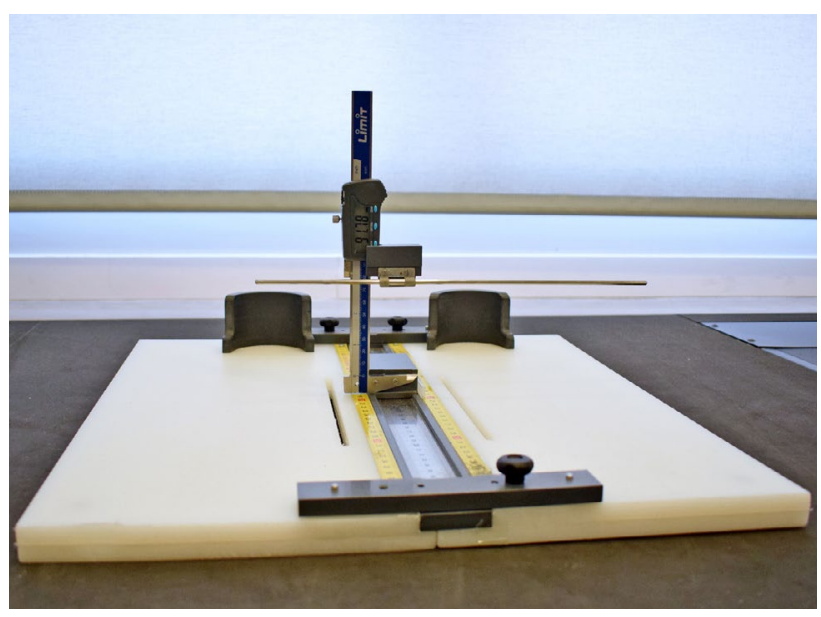

Figura 1. p-MAP.

metrada donde se observará la longitud del pie (Figura 1).

Para realizar las mediciones de altura del arco dorsal se utiliza un calibre (o pie de rey) digital con el brazo fijo, unido a una pequeña plataforma de 1,2 × 5,0 × 10,0 cm que lo mantiene vertical y que circula por la guía milimetrada. Presenta una barra horizontal, que se sitúa perpendicular al eje longitudinal pie, y que permite medir la altura del dorso del pie y también la longitud; para esta medida, se puede utilizar alternativamente un tope unido a una pequeña plataforma que circula por la guía.

Para medir la anchura del mediopié se utiliza un calibre (o pie de rey) digital, al que se la han añadido unas extensiones metálicas o de plástico en ambos brazos de 0,3 × 0,8 × 9,0 cm (Figura 2).

\section{Protocolo de valoración}

Se propone medir la movilidad vertical y latero-medial del pie, calculando la diferencia de estas medidas en carga y en descarga. Estudios previos han demostrado que ambas medidas tienen una gran fiabilidad y valide $z^{19,20}$.

El participante se sitúa sobre la plataforma, en bipedestación, con el peso repartido entre los dos pies al 50 \% (aproximadamente), con los brazos a lo largo del cuerpo y la mirada adelante. Se sitúan los pies con los talones en las "copas", lo más posteriormente posible, y las primeras cabezas metatarsales en contacto con el tope realizado a tal efecto (Figura 3a).

Dado que las medidas de longitud pueden representar variaciones importantes en función del tamaño del pie, se propone valorar el índice de la altura del arco (IAA, o Arch Height Index $[\mathrm{AHI}])$, dividiendo la altura dorsal entre la longitud total del pie. El resultado es un índice sin unidades que permite una comparación fiable entre pies de distintos tamaños.

El primer paso será obtener la longitud total del pie (la plataforma nos permite medir la longitud total o truncada [hasta

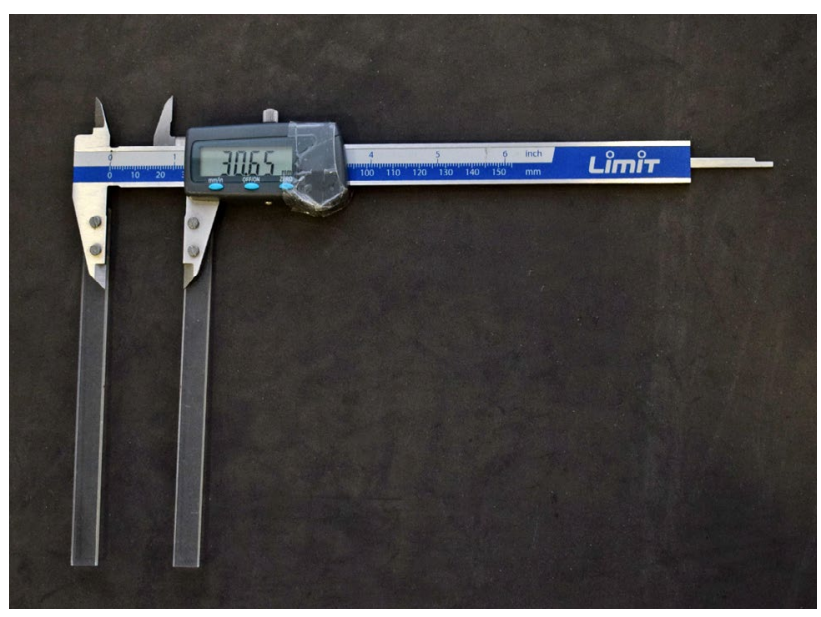

Figura 2. Calibre digital modificado para medir la anchura del mediopié.

la primera cabeza metatarsal] con el tope mencionado): para determinar la longitud total, se coloca el tope en el extremo más anterior de la plataforma y se desliza hasta que contacte con la punta del pie (normalmente el primer dedo). En este punto se observa la longitud en la guía milimetrada, se calcula la mitad de esta longitud y se realiza una marca en el dorso del pie con ayuda del calibre (Figura 3b).

Para valorar la altura del dorso del arco (ADA) se sitúa en primer lugar el calibre en la marca dibujada en el dorso y descendiendo la barra hasta el contacto con el pie, sin presionar los tejidos blandos. Para mejorar la fiabilidad de las medidas, se recomienda tomar la medida de la altura del arco dos veces en cada pie, y utilizar la media de ambas (Figura 3c).

Para valorar la anchura del mediopié (AMP), se sitúa el calibre en el $50 \%$ de la longitud total del pie (el mismo punto marcado para obtener la ADA) de forma perpendicular al pie, se hace contactar la parte fija (normalmente la izquierda) con el borde del pie (en contacto, pero sin presionar ni deformar los tejidos), y se desplaza la parte móvil cerrando el calibre hasta que esta también contacta con el borde opuesto del pie. Se puede comprobar que el contacto es correcto deslizando arriba y abajo los brazos del calibre, de modo que si hay demasiada presión no deslizará correctamente, y si falta el contacto se notará la holgura (Figura 4). Para una mayor fiabilidad, se recomienda tomar cada medida en dos ocasiones, y utilizar la media de ambas ${ }^{16}$.

Estas medidas corresponden a la postura del pie en carga. Para medir la movilidad del mediopié, se medirán a continuación los mismos valores en descarga situando al paciente en sedestación, con flexión de $90^{\circ}$ de caderas, rodillas y tobiIlos, y el pie en contacto con el suelo, pero sin más carga que el peso de la pierna, de forma similar al protocolo descrito para el test de caída del navicular (navicular drop test). Esta posición representa aproximadamente el $10 \%$ del peso del cuerpo $^{12}$.

De forma alternativa, y para eliminar totalmente el peso del 

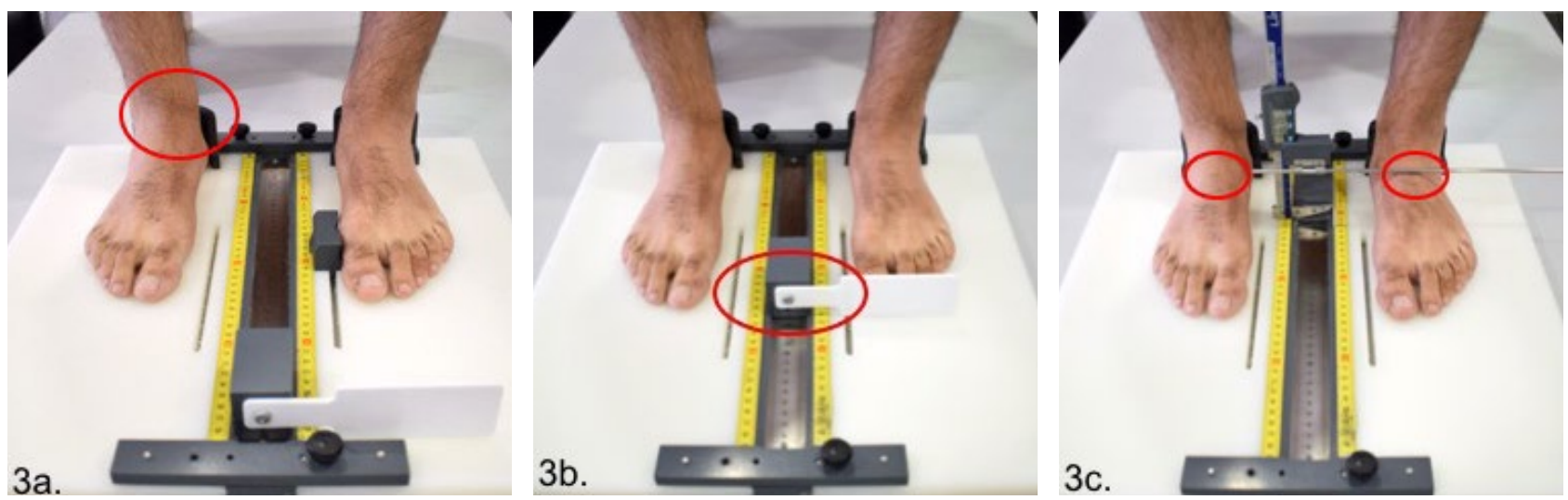

Figura 3. Descripción del proceso de medición mediante la p-MAP: a) colocación de los pies en la plataforma; b) medición de la longitud del pie; c) medición de la altura del arco en el $50 \%$ de la longitud del pie.

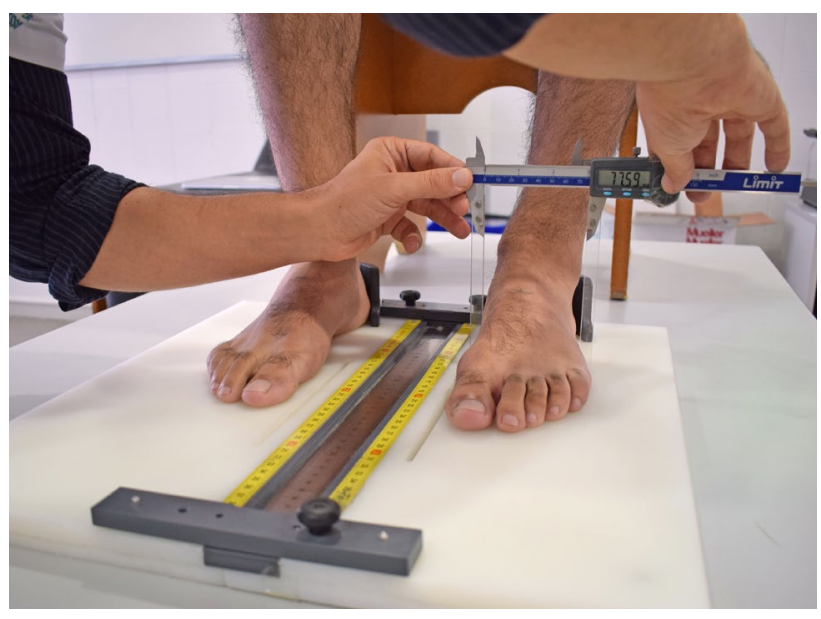

Figura 4. Medición de la anchura del mediopié mediante el calibre modificado.

cuerpo, McPoil y cols. diseñaron una plataforma específica para valorar la ADA en descarga, que se aplica al sujeto en sedestación con los pies colgando. Consiste en una plataforma portátil de plástico de 0,5 × 12,0 × 41,0 cm que el observador debe sujetar con una mano. Unido a esta plataforma se encuentra una guía por la que se desliza el calibre que medirá la altura del arco, adaptándose a las distintas longitudes del pie, del mismo modo que la plataforma fija del suelo. Para aumentar la sensación de contacto con la plataforma del participante en la planta del pie, se pegó un papel de lija de grano 80 a la superficie superior de la plataforma portátil ${ }^{16}$.

La principal limitación de esta medida es que requiere una plataforma específica y la colaboración del paciente para determinar el contacto con la superficie plantar, por lo que se recomienda realizar la medición en descarga con el pie apoyado en el suelo. La medición de la AMP en descarga total se puede realizar del mismo modo, con los pies colgando, aunque poniendo especial atención a que el calibre esté situado también de forma perpendicular en los tres planos, y desli- zando los brazos del calibre para comprobar que la anchura medida es la adecuada.

Para determinar la movilidad del pie en el plano sagital, se calculará la diferencia que presenta la ADA entre los valores obtenidos en carga y descarga, mientras que para obtener la movilidad en el plano transversal se utilizan las medidas de AMP. Una vez obtenidos los valores en carga y en descarga, se calcula la diferencia entre ambas medidas mediante una simple resta. Cuanta mayor diferencia exista entre las dos posiciones, mayor movilidad del pie ${ }^{21}$.

A través de los valores de movilidad en los dos planos (altura del arco y anchura del mediopié) podemos calcular la magnitud de movilidad del pie (MMP o Foot Mobility Magnitude [FMM]), que representa una medida compuesta de la movilidad del pie en estática. Se basa en el teorema de Pitágoras, según el cual, conociendo la longitud de dos de los lados de un triángulo rectángulo, se puede conocer la longitud del tercer lado; de este modo, conociendo las distancias vertical y latero-medial, podemos calcular la movilidad total del pie. Para ello, la diferencia de altura dorsal del arco (ADA) representa el lado vertical, mientras que la diferencia de la anchura del mediopié (AM) representa el lado horizontal; utilizando la fórmula citada, se puede calcular la hipotenusa, que corresponderá a la magnitud de movilidad del pie ${ }^{16}$.

$$
M M P=\sqrt{ }(A D A)^{2}+(A M)^{2}
$$

\section{Análisis estadístico}

Se ha comprobado la normalidad de las variables mediante la prueba de Kolmogorov-Smirnov y gráficos de probabilidad normal. Para las mediciones con, al menos, dos datos (datos en, al menos, dos artículos) se ha realizado una ponderación utilizando como pesos los tamaños de muestra del artículo respectivo. Se han calculado los intervalos de confianza del $95 \%$ para las medias resultantes ponderadas. El análisis gráfico ha consistido en diagramas de burbuja (bubble chart), 
donde el tamaño de la burbuja es proporcional al tamaño de la muestra (pesos). Se representan las medias y los tamaños de muestra para cada artículo.

Asimismo, se proponen unos valores de referencia para cada medida basados en las distintas muestras usadas para la ponderación. Se han calculado y representado los valores correspondientes a \pm 1DT (los cuales incluyen aproximadamente el $68 \%$ de la muestra) y \pm 1.5 DT (que incluyen al $86.6 \%$ ), como puntos de corte, en base a estudios previos ${ }^{16,22,23}$. Con la presentación de estas dos medidas, queda a elección del clínico la decisión de utilizar uno u otro valor como referencia.

Los valores de movilidad que se encuentren por debajo de 1 o 1.5 DT se podrán considerar pies rígidos o con una movilidad reducida, mientras que los que queden por encima se considerarán pies hipermóviles; estas medidas se realizan en dos planos perpendiculares (sagital y transversal) a través de los cuales se puede calcular una medida de la movilidad del pie global.

\section{RESULTADOS}

Se han analizado cinco artículos que suman un total de 1525 sujetos analizados. Las muestras estudiadas en el presente estudio corresponden a hombres y mujeres, pies derecho e izquierdo, de personas sin patología. Los tamaños totales de las muestras analizadas se muestran junto con su referencia en la Tabla I.

No todos los artículos analizados contienen todas las variables, en cuyo caso se indica el valor con la muestra total del artículo encontrado.

Todos los valores analizados, excepto uno (IAA), se miden en $\mathrm{mm}$, lo que los hace sencillos de comprender clínicamente: la altura del arco dorsal media para todas las muestras es de aproximadamente $64.5 \mathrm{~mm}$ en carga, mientras que aumenta a $74.2 \mathrm{~mm}$ cuando se elimina el peso y se sitúa al sujeto en descarga. En cuanto a la anchura del mediopié, los valores medios son de $83.5 \mathrm{~mm}$ en carga, que se reducen a $75 \mathrm{~mm}$ en descarga. En cuanto al valor de IAA, que no tiene unidades por lo que se puede comparar entre poblaciones con tamaños de pies muy distintas, el valor medio es de 0.251.

Los valores de referencia para la altura del arco (considerando que incluyen a un $86.6 \%$ de la población) son entre $56 \mathrm{~mm}$ y $72.9 \mathrm{~mm}$ en carga, y 66.7 y 86.8 en descarga. Teniendo en cuenta el IAA, los valores inferiores a 0,221 se pueden considerar pies planos, mientras que los mayores de 0.281 , serán pies cavos.

Los valores de referencia de la postura y la movilidad del pie basados en la bibliografía se detallan en las Tablas II y III, respectivamente, y se muestran para cada artículo estudiado en las Figuras 5 y 6 .

\section{DISCUSIÓN}

El presente trabajo describe el método de valoración de la postura y la movilidad del pie basado en la p-MAP (o Foot Assessment Platform, FAP, en inglés) e incluye el protocolo para su medición. Por otro lado, ofrece unos valores de referencia y puntos de corte basados en hasta 1525 sujetos, destacando el valor del Índice de la Altura del Arco de 0.251.

La postura del pie se puede definir como la forma que adopta este en posición estática, y su movilidad como la diferencia en una postura entre carga y descarga. Una de las característi-

\section{Tabla I. Referencia del artículo y tamaño de las muestras analizadas}

Artículo

número:

\begin{tabular}{cl} 
Art. 1 & $\begin{array}{l}\text { McPoil TG, Cornwall MW, Abeler MG, Devereaux KJ, Flood LJ, Merriman SE, et al. The optimal method } \\
\text { to assess the vertical mobility of the midfoot: navicular drop versus dorsal arch height difference. Clin } \\
\text { Res Foot Ankle. 2013;1:104. DOI: 10.4172/2329-910X.1000104 }\end{array}$ \\
\hline Art. 2 & $\begin{array}{l}\text { McPoil TG, Vicenzino B, Cornwall MW, Collins N, Warren M. Reliability and normative values for the foot } \\
\text { mobility magnitude: a composite measure of vertical and medial-lateral mobility of the midfoot. J Foot } \\
\text { Ankle Res. 2009;2(1):6. DOI: 10.1186/1757-1146-2-6. }\end{array}$ \\
\hline & $\begin{array}{l}\text { McPoil TG, Haager M, Hilt J, Klapheke J, Martinez R, VanSteenwyk C, et al. Can static foot posture } \\
\text { measurements predict regional plantar surface area? Foot. 2014;24(4):161-8. DOI: 10.1016/j. } \\
\text { foot.2014.07.003. }\end{array}$ \\
\hline Art. 4 & $\begin{array}{l}\text { McPoil TG, Cornwall MW, Vicenzino B, Teyhen DS, Molloy JM, Christie DS, et al. Effect of using } \\
\text { truncated versus total foot length to calculate the arch height ratio. Foot. 2008; 18(4):220-7. DOI: } \\
\text { 10.1016/j.foot.2008.06.002. }\end{array}$ \\
\hline Art. 5 & $\begin{array}{l}\text { McPoil TG, Warren M, Vicenzino B, Cornwall MW. Variations in foot posture and mobility between } \\
\text { individuals with patellofemoral pain and those in a control group. Am Podiatr Med Assoc. }\end{array}$ \\
\hline Total & 2011;101(4):289-96. DOI: 10.7547/1010289. \\
\hline
\end{tabular}




\begin{tabular}{|c|c|c|c|c|c|c|c|c|}
\hline Medidas & $\begin{array}{l}\text { Núm. } \\
\text { Artíc. }\end{array}$ & $\begin{array}{c}\mathrm{n} \text { total } \\
\text { (suma } \mathrm{n} \text { ) }\end{array}$ & Media & $\begin{array}{l}\text { Desviación } \\
\text { típica }\end{array}$ & $\begin{array}{l}\text { IC } 95 \% \\
\text { inferior }\end{array}$ & $\begin{array}{l}\text { IC } 95 \% \\
\text { superior }\end{array}$ & \multicolumn{2}{|c|}{$\begin{array}{l}\text { Cut-off points } \\
\pm 1 \text { DT } \pm 1.5 \text { DT }\end{array}$} \\
\hline Longitud del pie (mm) & 2 & 1195 & 256.52 & 17.0 & 228.90 & 284.13 & $\begin{array}{l}239.52 \\
273.52\end{array}$ & $\begin{array}{l}231.02 \\
282.02\end{array}$ \\
\hline Altura del arco en carga (mm) & 4 & 1439 & 64.46 & 5.62 & 63.67 & 65.24 & $\begin{array}{l}58.84 \\
70.08\end{array}$ & $\begin{array}{l}56.03 \\
72.89\end{array}$ \\
\hline Altura del arco en descarga (mm) & 3 & 589 & 74.23 & 5.02 & 63.11 & 85.35 & $\begin{array}{l}69,21 \\
79.25\end{array}$ & $\begin{array}{l}66.70 \\
86.76\end{array}$ \\
\hline Índice de la altura del arco (IAA) & 1 & 850 & 0.251 & 0.02 & $N D^{*}$ & $N D^{*}$ & $\begin{array}{l}0.231 \\
0.271\end{array}$ & $\begin{array}{l}0.221 \\
0.281\end{array}$ \\
\hline Anchura mediopié en carga (mm) & 2 & 397 & 83.46 & 6.28 & 78.82 & 88.10 & $\begin{array}{l}77.18 \\
89.74\end{array}$ & $\begin{array}{l}74.04 \\
92.88\end{array}$ \\
\hline Anchura mediopié descarga (mm) & 2 & 397 & 74.92 & 4.92 & 35.08 & 114.76 & $\begin{array}{l}70.00 \\
79.84\end{array}$ & $\begin{array}{l}67.54 \\
82.30\end{array}$ \\
\hline
\end{tabular}

*ND: no disponible por no poder realizar el cálculo con una sola referencia.

\begin{tabular}{|c|c|c|c|c|c|c|c|c|}
\hline Medidas & $\begin{array}{l}\text { Núm. } \\
\text { Artíc. }\end{array}$ & $\begin{array}{c}\mathrm{n} \text { total } \\
\text { (suma } \mathrm{n} \text { ) }\end{array}$ & Media & $\begin{array}{c}\text { Error } \\
\text { estándar }\end{array}$ & IC $95 \%$ & IC $95 \%$ & $\pm 1 \mathrm{DE}$ & $\pm 1.5 \mathrm{DE}$ \\
\hline Movilidad en altura del arco & 3 & 623 & 11.31 & 2.37 & 6.97 & 15.65 & $\begin{array}{c}8.94 \\
13.68\end{array}$ & $\begin{array}{c}7.75 \\
14.86\end{array}$ \\
\hline Movilidad anchura mediopié & 2 & 431 & 9.51 & 2.94 & 7.54 & 11.48 & $\begin{array}{c}6.57 \\
12.45\end{array}$ & $\begin{array}{c}5.10 \\
13.92\end{array}$ \\
\hline Magnitud movilidad del pie & 2 & 431 & 10.80 & 2.80 & -20.94 & 42.53 & $\begin{array}{c}7.90 \\
13.60\end{array}$ & $\begin{array}{c}6.60 \\
14.90\end{array}$ \\
\hline
\end{tabular}

cas del pie que se ha utilizado tradicional y actualmente por su relación con el riesgo de sufrir lesiones es su aplanamiento o altura del arco. La p-MAP permite valorar la altura del arco en la cara dorsal del pie, tanto en carga como en descarga. Esta medida se relaciona con diversos ángulos radiográficos que miden el aplanamiento del pie $\mathrm{e}^{16,21}$.

En el año 2000, Williams y McClay ${ }^{12}$ valoraron la fiabilidad de 7 pruebas clínicas para clasificar la postura del pie en dos situaciones: en descarga ( $10 \%$ del peso) y en carga monopodal (90\% del peso), que fueron: 1) altura del navicular (navicular height); 2) altura del arco dorsal (height of the dorsum of the foot); 3) ángulo del primer radio (angle of the first ray); 4) altura del navicular dividido entre la longitud del pie (navicular height divided by foot length); 5) altura del navicular dividido entre la longitud del pie "truncada" (navicular height divided by truncated foot length); 6) altura del arco dorsal dividida entre la longitud del pie (dorsum height divided by foot length), y 7) altura del arco dorsal dividida entre la longitud del pie "truncada" (dorsum height divided by truncated foot length). Entre todas ellas, concluyeron que la medida más fiable y válida fue la altura del dorso del pie dividida entre la longitud del pie, Ilamada Índice de altura del arco (IAA) (fiabilidad interobservador: $\mathrm{ICC}=0,811$ en $10 \%$ del peso, ICC $=0,848$ en $90 \%$ del peso; validez: ICC $=0,844$ en $10 \%$ del peso, ICC = 0,851 en $90 \%$ del peso).

McPoil y cols. ${ }^{24}$ introdujeron una modificación en el índice de altura del arco, repartiendo el peso de forma igual entre los dos pies (distribución del peso del 50 \% en cada pie). Con esta nueva característica, realizaron un amplio estudio con 850 sujetos (393 mujeres y 457 hombres), proporcionando unos valores recogidos en este estudio (Tabla II). Se valoró también su fiabilidad, mostrando una excelente fiabilidad intraobservador ( $\mathrm{ICC}=0,98$ a 0,99 y SEM $=0,03 \mathrm{a} 0,05 \mathrm{~cm}$ ) e interobservador $(\mathrm{ICC}=0,98$ a 0,99 y SEM =0,04 a 0,07 cm). Además, se estudió su validez mediante la comparación con las mismas medidas mediante radiografías (altura del arco dorsal y longitud del pie), con unos resultados que demostraron que no existían diferencias estadísticamente significativas entre las medidas clínicas y las radiográficas $(p<0,47)$.

En este estudio se valoró, asimismo, la relación entre la utilización de la longitud total del pie (clínicamente más sencillo de medir) y la longitud del pie truncado, que es como fue descrito por Williams y McClay. La conclusión fue que es posible realizar el IAA midiendo la longitud total del pie, siempre que no existan alteraciones digitales ${ }^{24}$.

En otro estudio, Franettovich y cols. confirmaron su fiabili- 

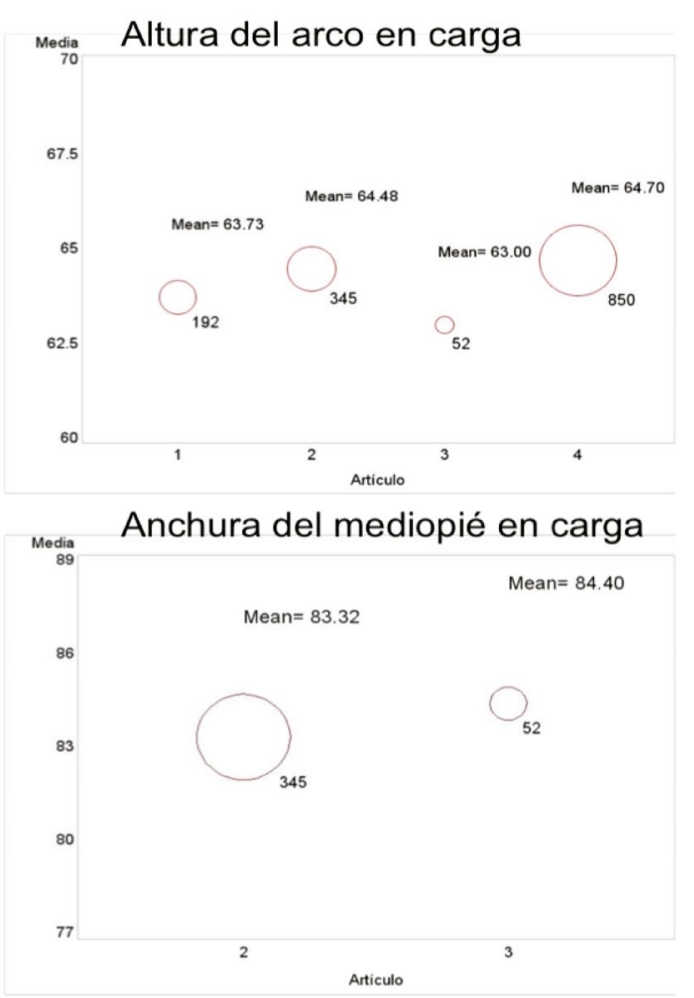
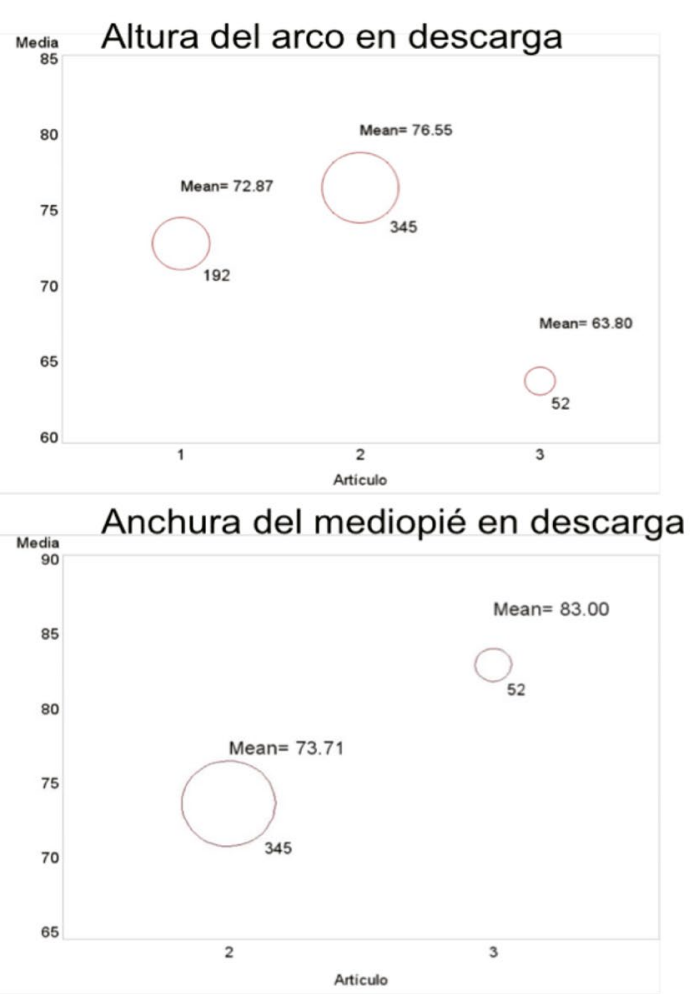

Figura 5. Media de la altura del arco y anchura del mediopié en carga y descarga, con el tamaño de la muestra (n) cuantificada y representada de forma proporcional según el radio de la burbuja.
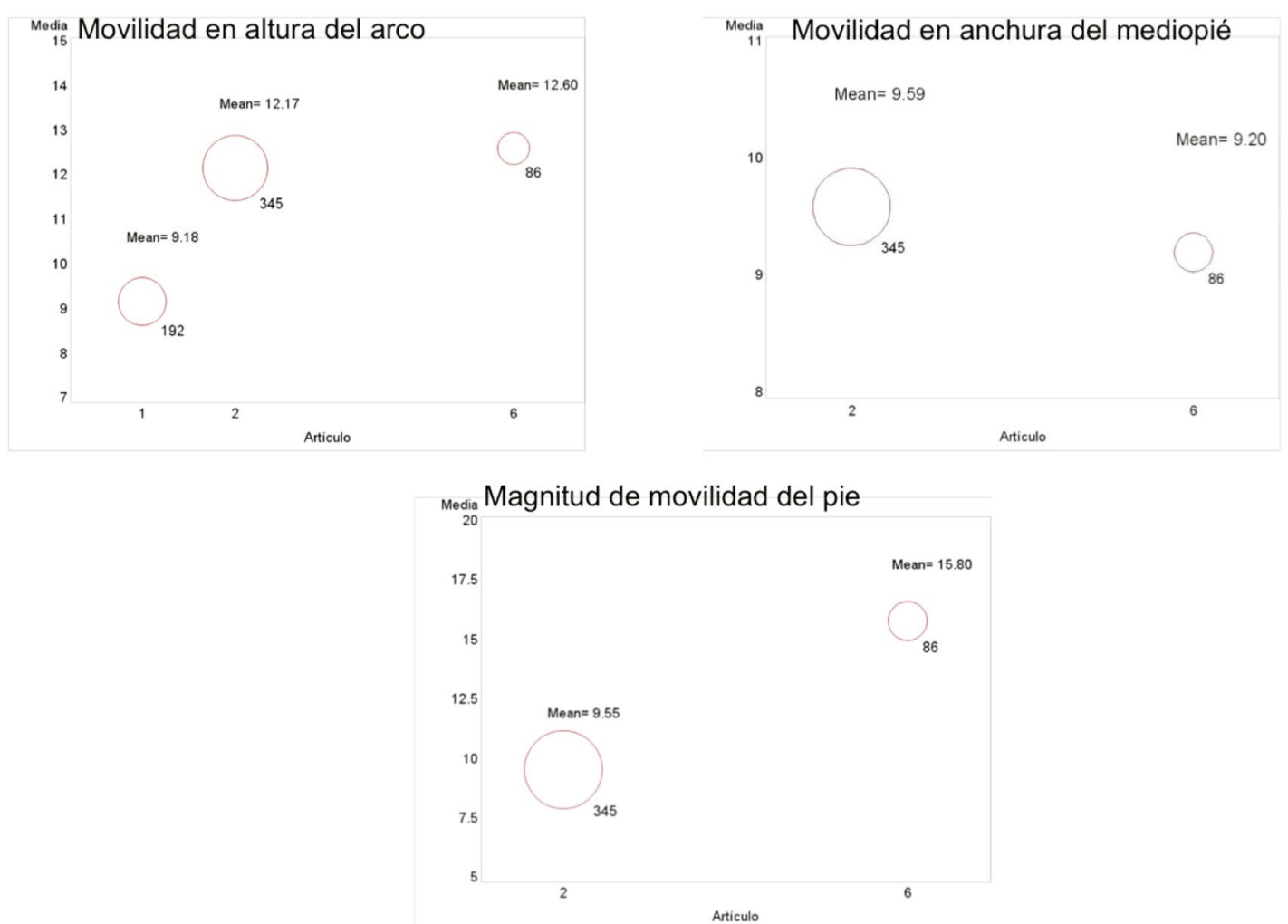

Figura 6. Media de la movilidad en altura del arco, anchura del mediopié y magnitud de movilidad del pie, con el tamaño de la muestra (n) cuantificada y representada de forma proporcional según el radio de la burbuja. 
dad y encontraron que, siendo una medida tomada en estática, parece predecir la postura del pie durante la marcha y la carrera (correlación $\left.r=0,85 ; r^{2}=0,72\right)^{25}$.

En cuanto a la movilidad del pie, un test habitual en la práctica clínica y en investigación es el test de caída del navicular (Navicular Drop Test, [NDT]). Propuesto por Brody en 1982 para evaluar la pronación del pie, se define como la diferencia en la posición (altura) de la tuberosidad del navicular en el plano sagital entre una postura neutralizada y relajada del $\mathrm{pie}^{26}$. Sin embargo, diversos estudios demuestran que el movimiento del navicular durante la marcha se produce tanto en una dirección vertical (cráneo-caudal) como horizontal (medio-lateral), por lo que un correcto análisis deberá incluir la movilidad en ambos planos ${ }^{27,28}$.

Para complementar el navicular drop test se describió el test de deriva o "patinamiento" del navicular (Navicular Drift Test), que valora el desplazamiento de la tuberosidad del navicular en el plano transversal, mediante una hoja de papel situada en el suelo. También en este caso se valora la diferencia entre el pie relajado y neutralizado, aunque con valores de fiabilidad solo moderados, y un elevado error estándar ${ }^{29}$.

En este artículo, se propone medir la movilidad vertical (en el plano sagital) y latero-medial (en el plano transverso) del pie mediante la p-MAP, a través de las cuales se puede calcular la movilidad global del pie. Todas estas medidas han demostrado su alta fiabilidad y validez en diversos estudios previos $^{10,16}$.

La diferencia en la altura dorsal del arco (DADA) es una medida de la movilidad del pie en el plano sagital. Es una alternativa al Navicular Drop Test, una valoración ampliamente utilizada, pero con unos niveles de validez y fiabilidad controvertidos. En cambio, los resultados de diversos estudios muestran unos altos niveles de fiabilidad y validez de la DADA ${ }^{21,30}$. Debe tenerse en cuenta que existe una baja correlación entre los valores del test de caída del navicular y la diferencia en la altura dorsal del arco (DADA), lo que indica que, aunque presentados como alternativa, valoran parámetros distintos ${ }^{20}$.

En cuanto a la medición de la anchura del mediopié (AMP), fue descrita por McPoil y Cornwall ${ }^{16,23,24}$ como alternativa al Navicular Drift Test, o a la prueba de patinamiento del navicular. Tiene el objetivo de cuantificar la movilidad en el plano transversal del pie a través del desplazamiento medio-lateral del mediopié. Una ventaja compartida respecto al Navicular Drop Test es que no requiere palpar y localizar la tuberosidad del navicular, ni de neutralizar el pie, los cuales pueden disminuir su fiabilidad.

En conclusión, en este artículo se describe el protocolo de medición para cuantificar de forma fiable y válida la postura (altura del arco y anchura del mediopié) y la movilidad del pie. La plataforma de medición antropométrica del pie (p-MAP) es una herramienta simple y de bajo coste que permite cuantificar el pie en cuanto a su postura (pies planos o cavos) y en cuanto a su movilidad. Además, permite calcular un índice normalizado a la longitud del pie (el índice de la altura del arco), que permite comparar pies de distintos tamaños. Los principales valores de postura del pie son una altura del arco dorsal de $64.5 \mathrm{~mm}$ en carga y $74.2 \mathrm{~mm}$ en descarga, una anchura del mediopié de $83.5 \mathrm{~mm}$ en carga y $75 \mathrm{~mm}$ en descarga, y un IAA de 0.251 . En cuanto a los valores de movilidad del pie, las medias son de $11.3 \mathrm{~mm}$ para la movilidad de la altura del arco y de $9.5 \mathrm{~mm}$ para la diferencia de anchura del mediopié. Los valores de normalidad (basados en 1.5 DE) se encuentran entre $56.0 \mathrm{~mm}$ y $72.9 \mathrm{~mm}$ en carga, y 66.7 y 86.8 en descarga para la altura del arco, y entre 0.221 (menor del cual se pueden considerar pies planos) y 0.281 (que corresponderían a pies cavos). Finalmente, la anchura del mediopié presenta unos puntos de corte de 74.0 y 92.9 en carga, y entre 67.5 y 82.3 en descarga.

\section{AGRADECIMIENTOS}

Los autores agradecen a la Direcció de Recerca i Innovació de la Universitat de Manresa (U. Vic - UCC) su ayuda y colaboración para la realización del presente artículo, especialmente a Jorge Ruiz y a Xavier Gironès.

\section{CONFLICTO DE INTERESES}

Los autores declaran que no existen conflictos de intereses sobre aspectos de financiación o de cualquier otro tipo.

\section{FINANCIACIÓN}

Ninguna.

\section{BIBLIOGRAFÍA}

1. Butler RJ, Crowell HP $3^{\text {rd }}$, Davis IM. Lower extremity stiffness: implications for performance and injury. Clin Biomech. 2003;18(6):511-7.

2. Riddle DL, Pulisic M, Pidcoe P, Johnson RE. Risk factors for Plantar fasciitis: a matched case-control study. J Bone Joint Surg Am. 2003;85$A(5): 872-7$.

3. Fong CM, Blackburn JT, Norcross MF, McGrath M, Padua DA. Ankle-dorsiflexion range of motion and landing biomechanics. J Athl Train. 2011;46(1):5-10. DOI: 10.4085/1062-6050-46.1.5.

4. Hernández-Gervilla O, Escalona-Marfil C, Corbi F. Relación entre la postura del pie y la cinemática de la carrera: estudio piloto. Apunts Medicina de I'Esport. 2016;51(192):113-148. DOI: 10.1016/j. apunts.2016.03.001.

5. Neal BS, Griffiths IB, Dowling GJ, Murley GS, Munteanu SE, Franettovich Smith MM, et al. Foot posture as a risk factor for lower limb overuse injury: a systematic review and meta-analysis. J Foot Ankle Res. 2014;7(1):55. DOI: 10.1186/s13047-014-0055-4.

6. McClay I, Manal K. Coupling Parameters in Runners with Normal and Excessive Pronation. J Appl Biomech. 1997;13(1):109-24. DOI: 10.1123/jab.13.1.109.

7. Williams DS $3^{\text {rd }}$, Davis IM, Scholz JP, Hamill J, Buchanan TS. High-arched runners exhibit increased leg stiffness compared to low-arched runners. Gait Posture. 2004 Jun;19(3):263-9. DOI: 10.1016/S09666362(03)00087-0.

8. Gross KD, Felson DT, Niu J, Hunter DJ, Guermazi A, Roemer FW, et al. Association of flat feet with knee pain and cartilage damage in older adults. Arthritis Care Res. 2011;63(7):937-44. DOI: 10.1002/acr.20431. 
9. Vicenzino B, Collins N, Cleland J, McPoil T. A clinical prediction rule for identifying patients with patellofemoral pain who are likely to benefit from foot orthoses: a preliminary determination. Br J Sports Med. 2010;44(12):862-6. DOI: 10.1136/bjsm.2008.052613.

10. Mills K, Blanch P, Dev P, Martin M, Vicenzino B. A randomised control trial of short term efficacy of in-shoe foot orthoses compared with a wait and see policy for anterior knee pain and the role of foot mobility. Br J Sports Med. 2012;46(4):247-52. DOI: 10.1136/bjsports-2011-090204.

11. Shultz SJ, Nguyen A-D, Windley TC, Kulas AS, Botic TL, Beynnon BD. Intratester and intertester reliability of clinical measures of lower extremity anatomic characteristics: implications for multicenter studies. Clin J Sport Med. 2006 Mar;16(2):155-61.

12. Williams DS, McClay IS. Measurements Used to Characterize the Foot and the Medial Longitudinal Arch: Reliability and Validity. Phys Ther. 2000;80(9):864-71.

13. Evans AM, Karimi L. The relationship between paediatric foot posture and body mass index: do heavier children really have flatter feet? J Foot Ankle Res. 2015;8:46. DOI: 10.1186/s13047-015-0101-x.

14. Cornwall MW, McPoil TG. Three-dimensional movement of the foot during the stance phase of walking. J Am Podiatr Med Assoc. 1999;89(2):56-66. DOI: 10.7547/87507315-89-2-56.

15. Lundgren $P$, Nester $C$, Liu A, Arndt A, Jones R, Stacoff A, et al. Invasive in vivo measurement of rear-, mid- and forefoot motion during walking. Gait Posture. 2008;28(1):93-100. DOI: 10.1016/j.gaitpost.2007.10.009.

16. McPoil TG, Vicenzino B, Cornwall MW, Collins N, Warren M. Reliability and normative values for the foot mobility magnitude: a composite measure of vertical and medial-lateral mobility of the midfoot. J Foot Ankle Res [Internet]. 2009;2(1): 6. DOI: 10.1186/1757-1146-2-6.

17. Bade MB, Chi TL, Farrell KC, Gresl AJ, Hammel LJ, Koster BN, et al. The use of a static measure to predict foot posture at midsupport during running. Int J Sports Phys Ther. 2016;11(1):64-71.

18. McPoil TG, Carrell D, Ehlers D, Kuhlman H, Mufti J, Pomeroy M, et al. Does foot placement affect the reliability of static foot posture measurements? J Am Podiatr Med Assoc. 2014;104(1):34-42.

19. Mills K, Blanch P, Dev P, Martin M, Vicenzino B. A randomised control trial of short term efficacy of in-shoe foot orthoses compared with a wait and see policy for anterior knee pain and the role of foot mobility. Br J Sports Med. 2012;46(4):247-52. DOI: 10.1136/ bjsports-2011-090204.

20. McPoil TG, Cornwall MW, Abeler MG, Devereaux KJ, Flood LJ, et al. The Optimal Method to Assess the Vertical Mobility of the Midfoot: Navicular Drop versus Dorsal Arch Height Difference? Clinical Research on Foot \& Ankle 2013;1:104. DOI: 10.4172/2329-910X.1000104

21. Escalona-Marfil C, McPoil TG, Mellor R, Vicenzino B. A radiographic and anthropometric study of the effect of a contoured sandal and foot orthosis on supporting the medial longitudinal arch. J Foot Ankle Res. 2014;7(1):38. DOI: 10.1186/s13047-014-0038-5.

22. McPoil TG, Cornwall MW. Use of the longitudinal arch angle to predict dynamic foot posture in walking. J Am Podiatr Med Assoc. 2005;95(2):114-20.

23. Cornwall MW, McPoil TG. Relationship between static foot posture and foot mobility. J Foot Ankle Res. 2011;4:4. DOI: 10.1186/1757-1146-4-4.

24. McPoil TG, Cornwall MW, Vicenzino B, Teyhen DS, Molloy JM, Christie DS, et al. Effect of using truncated versus total foot length to calculate the arch height ratio. Foot. 2008;18(4):220-7. DOI: 10.1016/j. foot.2008.06.002.

25. Franettovich MM, McPoil TG, Russell T, Skardoon G, Vicenzino B. The ability to predict dynamic foot posture from static measurements. J Am Podiatr Med Assoc. 2007;97(2):115-20.

26. Evans AM, Copper AW, Scharfbillig RW, Scutter SD, Williams MT. Reliability of the Foot Posture Index and Traditional Measures of Foot Position. J Am Podiatr Med Assoc. 2003;93(3):203-13.

27. Lundgren P, Nester C, Liu A, Arndt A, Jones R, Stacoff A, et al. Invasive in vivo measurement of rear-, mid- and forefoot motion during walking. Gait Posture. 2008;28(1):93-100. DOI: 10.1016/j.gaitpost.2007.10.009.

28. Cornwall MW, McPoil TG. Relative movement of the navicular bone during normal walking. Foot Ankle Int. 1999;20(8):507-12. DOI: 10.1177/107110079902000808.

29. Billis E, Katsakiori E, Kapodistrias C, Kapreli E. Assessment of foot posture: Correlation between different clinical techniques. The Foot. 2007; 17(2):65-72.

30. McPoil TG, Cornwall MW, Medoff L, Vicenzino B, Fosberg KK, Hilz D. Arch height change during sit-to-stand: an alternative for the navicular drop test. J Foot Ankle Res. 2009;2:17. DOI: 10.1186/1757-1146-1-3. 